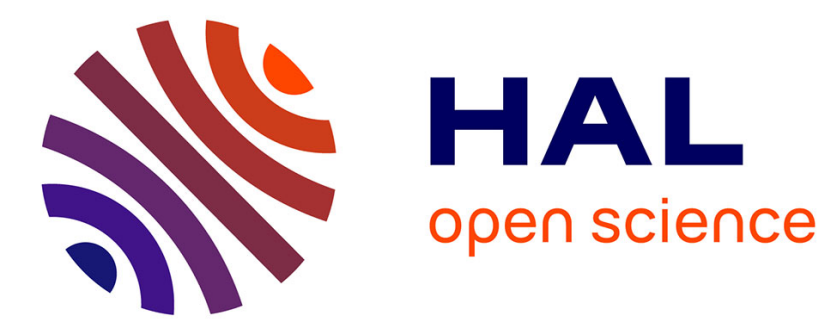

\title{
LES PHASES AMONT DES PROJETS INNOVANTS ET LA CONSTRUCTION DU GROUPE-PROJET
}

\author{
Iban Lizarralde, Véronique Pilnière
}

\section{To cite this version:}

Iban Lizarralde, Véronique Pilnière. LES PHASES AMONT DES PROJETS INNOVANTS ET LA CONSTRUCTION DU GROUPE-PROJET. International Journal of Projectics, 2012, 10 (1), pp.97110. hal-00735010

\author{
HAL Id: hal-00735010 \\ https://hal.science/hal-00735010
}

Submitted on 25 Sep 2012

HAL is a multi-disciplinary open access archive for the deposit and dissemination of scientific research documents, whether they are published or not. The documents may come from teaching and research institutions in France or abroad, or from public or private research centers.
L'archive ouverte pluridisciplinaire $\mathbf{H A L}$, est destinée au dépôt et à la diffusion de documents scientifiques de niveau recherche, publiés ou non, émanant des établissements d'enseignement et de recherche français ou étrangers, des laboratoires publics ou privés. 


\section{LES PHASES AMONT DES PROJETS INNOVANTS ET LA CONSTRUCTION DU GROUPE-PROJET Pour une approche socio-cognitive}

THE BEFOREHAND PHASES OF INNOVATIVE PROJECTS AND THE BUILDING-UP OF A PROJECT-TEAM Towards a socio-cognitive approach

LAS FASES DEL ESTABLECIMIENTO DE LOS PROYECTOS INNOVADORES Y LA CONSTRUCCIÓN DEL GRUPO-PROYECTO Hacia un enfoque sociocognitivo

\section{Lizarralde V. Pilnière}

\section{RÉSUMÉ}

Nous nous intéressons ici aux phases amont de la mise en place de projets complexes. Nous considérons comme « projets complexes », des projets qui se proposent de spécifier, de mettre en œuvre et de pérenniser des changements organisationnels profonds dans les entreprises ou, plus généralement, dans les activités sous contraintes économiques. Ces projets sont généralement liés à l'innovation sous ses diverses formes : innovation produit naturellement, mais aussi innovation process, innovation organisationnelle 
ou innovation sociale. Le manager ou le chef de projet en charge d'une telle transformation complexe va devoir réfléchir à la façon dont il va s'organiser, aux personnes concernées par le projet dont il prend la responsabilité. La question à laquelle nous allons nous intéresser dans cette contribution est la suivante : comment est-il possible de faire collaborer positivement des personnes et faire démarrer efficacement des projets communs dans la mesure où ces personnes défendent a priori des points de vue divergents sur la nature des problèmes posés, sur les solutions que l'on peut leur imaginer et sur des façons « réalistes » d'y parvenir.

Mots clés : complexité, changements organisationnels, innovation, socio-cognitique, projets.

\section{ABSTRACT}

In this paper, we deal with beforehand phases of complex projects. We consider as "complex" these projects which specify, implement and stabilize deep organizational changes in companies or more generally in the frame of activities under economic constraints. Such projects are generally linked to innovation, considered under its different states: product innovation of course, this contribution is as folbut also process innova- lows: how could we incention and organizational or social innovation as well. Project managers in charge of such a complex transformation will have to design the way he will organize his work, deeply taking in consideration the persons concerned by the project he his taking the responsibility. The question we are dealing with in tive persons to positively collaborate and make common projects start efficiently insofar as these persons a priori defend divergent points of view stake, on feasible solutions to these lasts and on "realistic" paths to achieve them? on the nature of issues at

Keywords: complexity, organizational change, innovation, socio-cognition, projects.

\section{RESUMEN}

Esta investigación se centra en las fases del establecimiento de un proyecto complejo. Consideramos un proyecto como complejo cuando en el seno de un proyecto se especifica, se implementa y se perenniza un conjunto de cambios organizacionales profundos dentro de la de este tipo de transempresa y de manera formaciones complejas general dentro de las acti- deberá reflexionar sobre vidades bajo restricciones la manera que se va a económicas. Estos proyectos están de forma general ligados a la innovación bajo diversas formas: evidentemente la innovación del producto, innovación organizacional e innovación social. El manager o jefe de proyecto al cargo la manera que se va a
organizar y a las personas involucradas en el proyecto de su responsabilidad. Este estudio pretende responder a la siguiente pregunta: Como se puede impulsar a las personas a colaborar de manera positiva y empezar proyectos 
en común de manera eficaz en la medida que estas personas defienden a priori puntos de vista divergentes sobre la naturaleza de los problemas expuestos, sobre las soluciones que creemos que imaginan y sobre las manera "realistas" de realizarlas.

Palabras claves: complejidad, cambio organizacional, innovación, socio-cognición, proyectos.

\section{INTRODUCTION}

Nous nous intéressons ici aux phases amont de la mise en place de projets complexes. Nous considérons comme « projets complexes», des projets qui se proposent, en tout cas dans l'ambition annoncée de leurs promoteurs, de spécifier, de mettre en œuvre et de pérenniser des changements organisationnels profonds dans les entreprises ou plus généralement, dans les activités sous contraintes économiques. Ces projets sont généralement liés à l'innovation sous ses diverses formes : innovation produit naturellement, mais aussi innovation process, innovation organisationnelle ou innovation sociale. Si tout changement peut difficilement être considéré comme une innovation, une innovation se conçoit difficilement, au moins dans sa dimension de diffusion, de déploiement ou de métabolisation dans la société ou, pour ce qui nous concerne ici, dans l'organisation, sans l'occurrence de changements organisationnels.

Dans cette mesure, nous entendons par changements organisationnels tous les changements qui prétendent faire en sorte que les personnes travaillent autrement, soient organisées ou s'organisent autrement pour travailler, pour coopérer, en général dans le sens d'une plus grande efficience ou de la mise en œuvre de modalités innovantes, au sens que nous venons de définir. Dans les contextes technico-économiques actuels, ces évolutions vont le plus souvent dans le sens d'une plus grande transversalisation des modes d'organisation, de la définition des processus clés du fonctionnement mis en œuvre pour l'activité récurrente ou de la mise en projet des activités non répétitives et vers l'aplatissement des modes hiérarchiques de commandement [ «tayloriens ») vers des modalités qui font une part croissante à l'empowerment des personnes et à leur responsabilisation. Comme nous l'avons souligné ci-dessus, ces évolutions peuvent se faire en liaison étroite avec des évolutions technologiques ou, au contraire, s'intéresser simplement à la mise en place de nouvelles formes d'organisation.

Le manager ou le chef de projet en charge d'une telle transformation complexe devra réfléchir à la façon dont il va s'organiser, à la « population » concernée par le projet dont il prend la responsabilité, et en particulier à la composition de l'équipe sur laquelle il va s'appuyer et à la façon dont il pourra s'y prendre pour faire avancer et partager un certain nombre d'idées ou de concepts qui lui semblent importants pour la réussite du projet. Il a également le souci d'inventer et d'organiser le management « flou » de ces phases amont 
qui précèdent le lancement formel du projet. Nous qualifions ces phases de «floues » dans la mesure où les processus de coopération qui s'y déroulent sont faiblement structurés et que la confrontation des points de vue des différents acteurs est essentiellement supportée par des échanges informels.

Mais, en même temps, le «travail » réalisé durant ces phases influence largement le devenir de ces projets d'innovation. Les arguments développés dans ces phases devraient conduire à la définition de spécifications propres (techniques, organisationnelles et économiques), servant d'éléments pour les spécifications d'un projet « officiel ». Et les modalités d'interaction de ces phases préalables, les opinions qui se forment durant celles-ci sont très lourdes de conséquences en ce qui concerne la « solidité » du futur groupe-projet. C'est surtout dans cette mesure que les phases amont sont vraiment très importantes.

La question à laquelle nous allons nous intéresser dans cette contribution est la suivante : comment est-il possible de faire collaborer positivement des personnes et de faire démarrer efficacement des projets communs (cette question nous positionne de fait sur la relation individu-groupe), dans la mesure où ces personnes défendent a priori des points de vue divergents sur la nature des problèmes posés, sur les solutions que l'on peut leur imaginer et sur des façons « réalistes » d'y parvenir. Ce changement des représentations des uns et des autres que cela suppose est fondamentalement un apprentissage.

Comme nous l'évoquions à l'instant, nous proposons un modèle qui s'intéresse aux « mécanismes » individu-groupe, en nous fondant sur une approche cognitive et organique de l'organisation apprenante. Nous tenterons d'analyser comment de telles divergences dans les appréciations et les motivations peuvent être surmontées dans le cas de projets opérationnels. En même temps, nous essaierons de comprendre comment le chef de projet peut prendre le leadership dans ces phases hautes des projets, afin d' « embarquer » le projet dans la « bonne » direction.

\section{L'IDENTIFICATION DU CHANGEMENT : UN « MELTING POT»}

Chaque individu génère dynamiquement ses propres représentations de son implication dans le projet dès que celui-ci « apparaît dans le paysage » en les « colorant » émotionnellement et en les chargeant de jugements de valeur (bien/mal, intéressant/banal, positif/négatif...). Un tel processus est lié à l'équation personnelle de chacun, à ses valeurs et croyances, à ce qu'il croit être ses intérêts (variés), à l'idée qu'il se fait de son image dans les yeux des autres, et de l'attirance relative (par rapport à d'autres polarisations cognitives) qu'il ressent pour l'entreprise, le département, le groupe, les personnes concernées et évidemment, le projet qui lui est proposé...

Mais en fait, l'organisation et « l'optimisation » se font toujours en « local». 
La recherche d'« holisticité » est certainement une de ces catégories cognitives qui sont en même temps souhaitables et non pertinentes, dans la mesure où il serait évidemment souhaitable d'envisager les choses dans toute leur complexité essentielle et relationnelle, mais qu'en même temps, nous sommes aussi évidemment incapables d'embrasser cognitivement toute cette complexité. Pour des raisons diverses, et de façon plus ou moins consciente, une personne impose toujours une clôture à ses ambitions cognitives : réalisme, pragmatisme, limites de « faisabilité » ou frontières cognitives impulsées par les routines, les règles, conventions, valeurs ou convictions... Dans la même ligne, c'est bien le cas concernant la question que nous posons en début de ce travail : la seule façon de travailler dans ce type d'environnement consiste à travailler sur des espaces d'intervention polarisés, correspondant à des champs cognitifs limités. En fait, une telle façon de travailler est nécessairement partielle et partiale, mais dans les phases préalables, il est très important d'imposer ces limites pour faciliter la polarisation positive des représentations des acteurs, dans l'optique de la constitution future du « groupe-projet ». Mais en même temps, il est également clair que si cette clôture est trop serrée, c'est la capacité du groupe concerné d'être effectivement innovant qui est en question! Quoi qu'il en soit, il est important de comprendre que ces limitations sont essentiellement de nature cognitive, car dans le monde « en vraie grandeur », ces aspects ignorés cognitivement travaillent « dans l'ombre » et feront tôt ou tard leur entrée en scène dans le champ de pertinence du projet et des acteurs.

Il est par ailleurs essentiel de travailler sur la nature complexe des liens entre individu et groupe. Notre conviction est que ce lien est le principal « melting pot » de la motivation et de l'apprentissage et, au fond, une des principales clés de la compréhension des phénomènes de changement. Il est donc important, pour le manager en particulier, d'approfondir cette approche pour mieux comprendre le phénomène projet : une microsociété faite d'individus et des individus faisant une microsociété. Le manager doit savoir travailler au niveau de ces groupes opérationnels. Tous les futurs acteurs du projet sont différents, tous ont des idées différentes et des projets individuels différents. Comment peut-on imaginer qu'ils se mettront facilement d'accord sur des objectifs communs, sur la façon de travailler ensemble, comme s'il n'y avait pas de divergences de représentations, d'opinion ou d'intérêts, ni de problèmes «politiques » (au sens des problèmes de pouvoir) ? Dans cette optique, encore une fois, les phases liminaires présentent un intérêt majeur.

\section{AUTOUR DU CONCEPT DE GROUPE (DE PROJET)}

Quelle est donc la « réalité » d'un groupe ? Est-ce plus qu'une collection d'individus ? Quelle est sa solidité monolithique, sa grégarité, sa capacité à générer un magnétisme interne?

Le simple fait de poser ces questions met en question l'attitude cognitive qui consiste à considérer le groupe ontologiquement, comme une sorte de rouage ou un objet compact, et qui conduit à ignorer la nature complexe du 
groupe. Une telle simplification aura un impact sur les processus concernés car les résultats concrets réalisés par le groupe en action ne seront certainement pas ceux que l'on escomptait à partir d'une telle vision mécaniciste du fonctionnement du groupe. Cette question est omniprésente dans l'entreprise, chaque fois en tout cas que l'on s'y préoccupe de la mise en œuvre ou du " déploiement », de ce fameux écart entre la décision et ses effets concrets. C'est la fameuse question du « pourquoi les salariés ne font pas ce que l'on attend d'eux ? » que les managers connaissent bien au moment de mettre en application les décisions prises ! Un groupe peut-il être (impunément) compris comme un tout homogène, comme une « boîte noire » ou un « rouage » dont le comportement serait prévisible de l'extérieur, voire « pilotable » selon des plans définis de façon externe ou hiérarchique?

Si la réponse est oui (comme beaucoup de travaux de recherche et de pratiques d'entreprise le supposent, plus ou moins consciemment), on arrive à considérer (encore une fois, plus ou moins consciemment) que le groupe est suffisamment compact pour être représenté par son leader ou son chef et assimilé à lui, notamment vis-à-vis de ses partenaires externes (mais aussi d'ailleurs vis-à-vis de ses membres). Dans ce cas, un groupe est envisagé comme un rouage d'une organisation structurée verticalement. Il est compris comme une entité à comportement prévisible ou en tout cas « pilotable ». Cette optique est en parfait accord avec les approches néo-tayloriennes et mécanistes de l'organisation, en accord avec une Weltanschauung qui considère qu'un système humain ou anthropotechnique est susceptible d'être conduit comme peut l'être une machine. Cette vision est congruente avec une conception « légitimiste » de l'organisation, selon laquelle il est dans la nature des choses que le chef dirige, que les subordonnés exécutent et que la boucle se ferme par le contrôle de l'exécution. Selon cette optique, un tel système doit fonctionner. Si ce n'est pas le cas, alors il s'agit, pour ces esprits légitimistes, d'un dysfonctionnement pathologique et il est de la responsabilité du chef de trouver une solution. Celle-ci reste bien sûr la plupart du temps dans le même paradigme.

L'expérience de terrain, mais aussi la littérature sur le sujet (de Lewin ${ }^{1}$ à Argyris $^{2}$, de Crozier $^{3}$ à Morin ${ }^{4}$, en passant par Latour ${ }^{5}$ et Boltanski et Thévenot $^{6}$ l poussent à douter de la légitimité et de l'efficacité d'une telle position scientifique. Il faut avancer dans la réflexion épistémologique sur le concept de groupe. La première question concerne le terme de « groupe », piège lan-

1. Hothersall, David (1995). History of Psychology. New York: McGraw-Hill, pp. 239-253.

2. Argyris Chris (1993), Knowledge for action: A guide to overcoming barriers to organizational change. San Francisco, CA: Jossey-Bass Publishers.

3. Crozier, Michel (1977). L'acteur et le système, Paris, Le Seuil.

4. Morin, Edgar (1997). Réforme de pensée, transdisciplinarité, réforme de l'Université, Communication au Congrès International «Quelle Université pour demain ? Vers une évolution transdisciplinaire de l'Université » (Locarno. Suisse, 30 avril-2 mai 1997) ; texte publié dans Motivation, ${ }^{\circ}$ 24, 1997.

5. Latour Bruno (1991). Nous n'avons jamais été modernes. Essai d'anthropologie symétrique. Paris, La Découverte.

6. Boltanski, Luc et Thévenot, Laurent (1989). « Justesse et justice dans le travail », Cahiers du centre d'études de l'emploi, Paris, Presses universitaires de France. 
gagier au sens de Michel Foucault ${ }^{7}$, qui par sa simple existence crée une catégorie cognitive, qui donne ipso facto une présomption d'existence ontologique à cette substance qu'il est supposé dénoter. La piste qui se déduit de cette première réflexion est d'aller questionner la complexité qui se cache derrière ce mot, notamment la place des individus et de leur activité cognitive et pratique dans la « construction » d'un groupe... Il est important de s'arrêter un moment sur cette réflexion.

\section{INDIVIDUS ET GROUPES : UN MODËLE ORGANIQUE ET COGNITIF}

« L'existence » d'un groupe est donc fortement associée aux constructions cognitives que les personnes s'en font. Elle appelle donc une réflexion sur la nature fondamentale de ce qu'est le groupe et sur le double lien individu/ groupe. Le groupe apparaît essentiellement comme une figure présente dans un ensemble de représentations mentales où chaque individu du groupe conçoit qu'il fonctionne en groupe, le valorise positivement et redouble donc de production de liens et d'échanges avec les autres membres du groupe. C'est un tel processus (processus auto-poïétique) qui va entraîner le développement de la densité du groupe, en même temps que la prise de conscience par chacun de cette densité et du fait que les autres font en quelque sorte « allégeance » au groupe va autoalimenter le processus. Les représentations (en particulier celles liées au groupe, au projet) sont donc en construction continue, irréductiblement individuelles, intimes même (certains auteurs parlent de connaissance tacite ${ }^{8}$ ) et en même temps groupales, car elles sont forcément liées au « monde réel » qui est fait, entre autres choses, des autres personnes du groupe. Elles se développent, se nourrissent et se valident dans des réseaux, des échanges et des relations qui sont précisément la substance des groupes, et notamment des groupes-projets. Dans ces conditions, l'importance des phases amont se fait bien évidente. À noter aussi que nous focalisons ici sur le fonctionnement du groupe vu « de l'intérieur », mais qu'un raisonnement voisin pourrait être mis en œuvre, à propos de la représentation du groupe chez les personnes qui n'en font pas partie.

En fait, dans la perspective de la dynamique des projets, tant sur le plan de la constitution des groupes que de l'innovation et des apprentissages qu'y peuvent s'y dérouler, ces questions apparaissent comme centrales, dans la mesure où l'on prétend réfléchir aux (bonnes) façons de manager de telles pratiques. À souligner cependant que ce qui s'échange dans de tels réseaux n'est pas de la connaissance mais de l'information?. La connaissance au sens strict est à notre sens un construit cognitif. Dans cette mesure, la connaissance

7. Michel Foucault (1966). Les Mots et les Choses, Archéologie des sciences humaines, Paris, Gallimard, NRF.

8. Nonaka, Ikujiro \& Takeuchi, Hirotaka (1995), The Knowledge-Creating Company, Oxford University Press.

9. Specialists in Information Systems make a distinction between data and information. We will here consider theses two words as synonymous. Our distinction will be made between, on the one hand, these two terms (a raw message on a support) and, on the other hand, the term of knowledge (an interpreted notion, hence cognitive processes proper to the individual). 
doit être considérée comme construite dans la tête de chacun, mais en même temps, elle est toujours construite dans le contexte de processus collectifs.

De ce point de vue, un modèle organique du groupe est nécessaire pour rendre compte du lien entre les deux niveaux ci-dessus définis. Chaque individu y est considéré comme une « unité cognitive », produisant des représentations et fonctionnant dans un processus continu d'interaction avec les autres individus composant le réseau et de perception d'informations internes ou externes au réseau. L'activité cognitive de chacun des individus est fonction d'éléments plus ou moins stables (culture, valeurs, intentions stratégiques, éléments mémorisés...) et d'éléments plus ou moins volatils (perceptions, contextes et intentions à court terme, émotions...). Tous ces éléments en interaction dans une sorte de cycle perception/représentation/action produisent un perpétuel processus d'émergence. Chaque individu agit sans lien « mécanique » vers les autres ou vers sa hiérarchie : comme individu, il est doté fondamentalement d'une marge d'autonomie, quels que soient les principes de comportement, les règles, les coordinations et autres injonctions hiérarchiques plus ou moins imposés, acceptés, voire même autoproduits... et finalement institutionnalisés dans l'environnement.

Le « niveau collectif »- mais qui n'est autre que l'ensemble des interactions en œuvre (coopération, coordination) et des représentations de celles-ci et donc du groupe qui se construisent (ou se déconstruisent) - doit être considéré comme émergeant de ces réseaux dynamiques complexes et à son tour comme exerçant un impact sur le niveau individuel, dans la mesure où son émergence change le contexte court terme et donc a un impact sur les perceptions et l'activité cognitive des individus. Toutes ces dynamiques génèrent en outre des apprentissages, puisqu'elles changent les valeurs, la « culture locale » et les représentations des individus et, par la même occasion, leur « équation personnelle » (ce point renvoie aux théories de Goffman ${ }^{10}$ et Bateson ${ }^{11}$, ainsi qu'aux théories de l'institution ${ }^{12}$, des conventions ${ }^{13}$, de la stratégie « chemin faisant ${ }^{14} \ldots$...

\section{LA DIMENSION COGNITIVE (... UNE AUTRE VISION DE LA «GESTION DES CONNAISSANCES »)}

Dans cette contribution, notre attention est focalisée sur ce que ce modèle peut apporter à la théorie des phases amont des projets complexes. Que se

10. Goffman, Erving (1959). The Presentation of Self in Everyday Life. New York: Doubleday.

11. Bateson, Gregory (1973). Steps to an Ecology of Mind. Paladin Books.

12. Lourau, René (1990). La politique de la surimplication, Technologies et Symboliques de la Communication, sous la direction de Lucien Sfez et Gilles Coutlée, avec la participation de Pierre Musso. Presses universitaires de Grenoble

13. Orléan, André (Éd.), (2000). Analyse économique des conventions, Paris, PUF.

14. Avenier, Marie-José (Éd.) (2000). Ingénierie des pratiques collectives. La cordée et le quatuor, Paris, L'Harmattan, coll. « Ingenium ». 
passe-t-il dans ces phases précoces ? Comment peut-on admettre sans discussion que les individus concernés vont converger automatiquement dans leur appréhension des problèmes et dans leur capacité à construire des actions et des compétences collectives efficientes? Pour les raisons exposées ci-dessus, nous pensons qu'une telle construction ne peut se réaliser que collectivement et dans l'action. Chacun doit y trouver quelque chose, au moins au niveau des représentations qu'il se fait de son inscription dans les groupes concernés.

Notre idée est donc d'adapter le modèle ci-dessus défini aux environnements en changement et en apprentissage et de comprendre quels sont les principaux enjeux des phases amont des projets innovants. Quelles sont les compétences comportementales que les acteurs doivent développer et de là, quelles sont les pratiques de management qui doivent être favorisées ? Cette question suppose que l'on approfondisse la compréhension de tels phénomènes liés aux groupes-projet, notamment dans les phases précoces de ceux-ci.

Il ne fait pas de doute que la connaissance est un phénomène personnel et intime. Elle est mouvante et est souvent mise en mouvement par le contact (médiatisé ou non) avec les autres. Les stimuli reçus (perception de signes) ont la propriété de déclencher des processus de construction de connaissances dans le cerveau de tout un chacun. La construction de connaissance doit être considérée comme un « maelström » cognitif intime, mais qui fonctionne fondamentalement en relation avec ses propres actions et les actions et le fonctionnement cognitif des autres. Le travail et l'apprentissage collectifs peuvent se comprendre comme des « réseaux de cerveaux » en (inter-laction dans des conditions concrètes et situées, ces dernières offrant en même temps des opportunités et des contraintes (« l'enaction » de Varela et Maturana ${ }^{15}$ ).

Dans ce mouvement, l'individu produit des représentations sur son environnement, sur les accords qui s'y produisent, sur la cohérence de ce qui s'y passe, le tout en rapport avec ses propres valeurs et sa propre culture... II essaiera parfois de mieux comprendre les problèmes des autres, il sera plus réceptif aux idées des autres, il sera ouvert à l'évolution de ses représentations sur son rôle et sa place dans le groupe, pour tenter de contribuer à un changement positif. Ou à l'opposé, il focalisera sur des aspects négatifs, sur des malentendus, des conflits d'intérêts, des inimitiés... Selon la « coloration » qu'il attribuera à ces relations en construction, il construira des représentations positives sur l'intérêt de ce qu'il fait et se laissera happer par l'aventure du projet, de l'amélioration, du travail créatif et de l'apprentissage ou, au contraire, il restera sur la réserve, développera une attitude hostile... Toujours dans les dynamiques collectives dont nous avons fait état ci-dessus. Le choc instable de tous ces éléments, mais aussi de toutes les représentations que les individus s'en construisent, est à la base de la performance collective.

Comment est-il possible que des personnes munies de Weltanschauungen, porteuses de différents projets personnels, d'intérêts différents voire antagoniques, puissent travailler, s'impliquer et créer positivement et efficacement ensemble? Certainement pas par hasard!

15. Maturana, Humberto \& Varela, Francisco (1980), « Autopoiesis and Cognition: The Realization of the Living », Boston Studies in the Philosophy of Science, Cohen, Robert S., and Marx W. Wartofsky (Eds.), Vol. 42, Dordecht: Reidel Publishing Co. 


\section{MANAGER LES PROJETS D'INNOVATION?}

Cette problématique est d'entrée paradoxale car associer deux logiques qui paraissent antagoniques, la logique de la « gestion », plutôt une logique d'ordre et d'obéissance à la règle, et la logique d' « innovation », plutôt une logique de désordre et de rébellion, peut sembler une gageure. Deux solutions opposées, extrêmes, s'offrent au manager : « forcer » le changement selon un plan imposé ou, au contraire, travailler à la construction d'espaces de collaboration positifs. Mais tenter de «forcer » le système, c'est faire bon marché de sa complexité et de la nécessaire autonomie des acteurs (le système ne peut fonctionner sans ellel et donc, par la même occasion, des marges de manœuvre - immenses! - que les acteurs possèdent pour ne pas se soumettre, voire pour marcher en sens inverse ! Il est clair que cela ne marchera pas.

En « forçant le trait », deux méthodes s'offrent au manager : «forcer » la cohésion et la grégarité du groupe, en focalisant sur un plan imposé, ou à l'inverse, travailler sur l'émergence d'espaces partagés de collaboration.

- Nous pensons que tenter de «forcer » le système revient à faire bon marché de la complexité de celui$c i$ et de l'autonomie des acteurs. Dans des environnements orientés vers les projets d'innovation, demandant à la créativité de s'exprimer, l'autonomie des acteurs est majeure. Il est impossible de mobiliser les intelligences et de les rendre responsables et créatives sans s'éloigner des modes cartésiens et tayloriens classiques.

- L'autre méthode, fondée sur l'autonomie, la confiance et l'auto-organisation présente cependant le risque évident de voir les énergies se disperser en ne rencontrant pas de focalisation en commun et en générant des situations conflictuelles. Faute de construire une polarisation ou en cas de développement d'une polarisation négative, cette autonomie peut certainement conduire le groupe au chaos et à l'insubordination, en regard des projets du manager en tout cas.

Le chemin est donc étroit pour le manager : il consiste à naviguer à vue entre ces deux pôles, dans l'optique de faire évoluer et se rapprocher les positions des uns et des autres, de «traduire » (au sens de Latour et Callon) ${ }^{16}$, c'est-à-dire de faire évoluer, par l'action collective, les représentations qui sont dans le jeu, en générant des représentations plus positives, plus « solidaires » et plus flexibles. Il faut arriver à ouvrir les acteurs au changement et à intégrer à ces représentations le fait que la route qui s'ouvre avec le groupe projet est intéressante justement parce qu'elle apporte de nouvelles idées et façons de fonctionner. Le chef de projet, ou la personne en charge du

16. Callon Michel (1986). "Éléments pour une sociologie de la traduction. La domestication des coquilles Saint-Jacques et des marins-pêcheurs dans la baie de Saint-Brieuc », L'Année sociologique, vol. 36, pp. 169-208. 
groupe, doit comprendre comment fonctionne la dialectique complexe individu/groupe en intériorisant le modèle que nous proposons ici. C'est la condition de la convergence des énergies individuelles vers l'efficience du travail coopératif ${ }^{17}$.

La constitution d'un groupe en charge de l'innovation demande donc des interventions de «l'ordre du discours », pour reprendre l'expression de Michel Foucault, mais aussi de travailler au niveau opérationnel. Le développement de l'identité du groupe est lié aux représentations qui « instituent »le groupe comme un lieu où l'on fait quelque chose d'intéressant et de valorisant, où l'on vise l'atteinte d'un objectif matérialisable, suffisamment concret pour qu'on puisse le voir se construire et progresser. À cette institutionnalisation en positif du groupe est lié l'approfondissement des sentiments d'appartenance. C'est la seule façon de permettre aux énergies de se mettre en synergie et de permettre la coopération en transcendant les différences et les conflits idéologiques, interpersonnels, intermétiers, voire interculturels. S'en tenir à un niveau générique, fonctionnant sur de la communication abstraite, du seul ordre du discours, n'est qu'une sorte d'imprécation et conduit le plus souvent à l'échec. Dans ce cas, en effet, les différences et conflits à l'instant évoqués lils sont de l'ordre de l'idéologie, donc d'une certaine façon de l'ordre du discours) réapparaîtront rapidement, dans la mesure où ils ne seront pas transcendés par le challenge concret du faire, par la réalisation d'une chose anticipée comme digne d'intérêt. Au-delà, le manque de concret et de choses «montrables » permet aux opposants de convaincre les indécis, dans la mesure où il n'y a pas de réalisation concrète permettant de contrer ces tendances centrifuges en montrant «qu'il se passe quelque chose » d'intéressant dans le groupe projet.

Nous sommes certainement en présence des fondations en profondeur du « learning by doing » des théoriciens anglo-saxons de l'apprentissage. Pour les raisons que nous avons évoquées, l'action située est un facteur important de la plasticité des représentations mentales. Centrer les intérêts des acteurs en essayant d'imposer des «clôtures » cognitives qui « valorisent » le groupe projet, peut-être même qui le « survalorisent », est sans doute la seule façon d'aider les uns et les autres à se construire une consistance cognitive (une existence « attestée ») pour le projet qui débute. Cela est nécessaire pour qu'ils puissent s'y imaginer leur futur rôle et générer « du lien » avec les autres acteurs de l'aventure, c'est-à-dire ni plus ni moins, comme nous l'avons expliqué dans notre modèle, pour qu'ils puissent commencer à construire le groupe. Une telle ambition doit à l'évidence être gérée !

Le manager, ou la personne qui prétend démarrer un projet en lui donnant une certaine direction, doit donc jouer un jeu subtil. Il doit naviguer entre l'acceptation de dynamiques émergentes (la coopération) et les règles qu'il essaiera d'imposer (la coordination). Savoir dominer ce jeu complexe est fondamentalement le talent du bon manager, notamment dans les phases hautes des projets, talent qui fait sans doute la différence avec un chef de projet de moins bon niveau. Même si à l'évidence les aspects « durs » de la gestion

17. « Le groupe performant est non seulement celui qui définit des stratégies appropriées à son contexte, mais encore celui qui est capable de mobiliser l'ensemble des énergies et des compétences nécessaires à l'approche de ses objectifs ». Nicole Fontaine, ministre française de l'Industrie, in Industries, n 84, mars 2003. 
de projets doivent être gérés, nous pensons, dans la mesure où c'est fondamentalement la génération de liens étroits entre les personnes qui « fait » le groupe-projet, que l'ensemble des aspects « soft » est très important. Tout cela est bien de l'ordre des représentations mentales.

Plusieurs caractéristiques devront donc être mises en œuvre, voire imposées (avec tact et modération) dans les phases hautes :

- Des moyens pour les échanges internes au groupe en gestation doivent être mis en œuvre, et la densité de ces échanges doit également être soutenue, de façon à faire en sorte que les acteurs concernés focalisent effectivement sur le projet concerné.

- Un autre enjeu concerne la capacité collective à prendre la même route. Au-delà des divergences, il est important de favoriser le rapprochement des représentations des membres du groupe à propos du « où allonsnous? » ou du «pour qui travaillons-nous? ». Dans cette mesure, les phases hautes sont certainement le germe de la «culture projet » et au-delà de « l'identité projet », source du développement des sentiments d'appartenance, au moins dans l'esprit des membres du groupeprojet, mais certainement aussi vis-à-vis des personnes extérieures.

- D'autre part, le fait de travailler sous la pression (du temps notamment) est certainement un catalyseur de l'action collective (le « drill » de Bruno Latour ${ }^{18}$ ).

L'apprentissage est fondamentalement un changement dans les représentations. Le modèle que nous proposons permet de mieux comprendre de ce qu'un (futur) manager doit prendre en considération lors de ces phases amont des projets d'innovation. Ce modèle est plat ll'organisation est « locale en tout point $\gg{ }^{19}$ ), organique (des individus en réseaux) et cognitif (chaque individu est considéré comme une «centrale cognitive», producteur de représentations, pensant, « computant » et agissant, en entendant ces processus selon le modèle des boucles sensori-motrices). L'intervention des managers dans ces environnements (ainsi d'ailleurs que celle des chercheurs) doit prendre en compte ces deux aspects en les interconnectant : individu et liens interpersonnels (quelque chose comme le «chemin de longueur un » de la théorie des graphes).

Pour reprendre le beau poème de Machado, « le chemin se fait en marchant », et la phase de départ, les premiers pas sur le chemin d'un projet d'innovation constituent à l'évidence une phase très importante. L'intention stratégique définit, du point de vue du management, «l'espace d'acceptation » dans lequel les idées et contraintes fondamentales doivent être intégrées. L'enjeu étant de trouver le moyen, les modalités pour que cet espace soit intégré en tant que tel dans les représentations des acteurs.

18. Latour, Bruno, ibid.

19. Latour, Bruno (1991), Nous n'avons jamais été modernes. Essai d'anthropologie symétrique. Paris, La Découverte. 


\section{CONCLUSION}

Le management doit être en phase avec les enjeux de l'apprentissage organisationnel. Mieux saisir la nature intime de la connaissance aide à comprendre comment savoir et apprendre sont liés aux émotions, aux représentations et aux opinions que les acteurs se forgent en relation aux projets d'innovation où ils sont susceptibles d'être immergés. La motivation et la construction du groupe ne peuvent que s'améliorer si l'action donne des résultats concrets et si les personnes se forgent des représentations qui « font sens » (signification, direction et réalisation personnelle). Ceci est très important au regard de « l'utilité sociale » que chacun ressent dans ses représentations autoréférentielles : cette impression que « ce que je fais est utile », ou qu' « ils sont intéressés par ce que je dis ou je fais », ou bien encore que «j'éprouve un réel intérêt à travailler à telle ou telle tâche avec un tel ou une telle »...

Pour nous, «piloter » le groupe dans les phases hautes signifie essentiellement que le (futur) manager essaie d'interférer dans les processus cognitifs des membres du groupe en gestation, en tentant de leur offrir des espaces de construction de sens, d'identité et de représentations positives en relation au projet qu'il veut lancer. Ce qui est en jeu, c'est une atmosphère constructive et des sentiments positifs d'appartenance ou, à l'opposé, une atmosphère attentiste, du scepticisme et du conflit...

\section{BIBLIOGRAPHIE}

Argyris Chris (1993), Knowledge for action: A guide to overcoming barriers to organizational change. San Francisco, CA: Jossey-Bass Publishers.

Avenier, Marie-José (Éd.) (2000), Ingénierie des pratiques collectives. La cordée et le quatuor, Paris, L'Harmattan, coll. «Ingenium ».

Bateson, Gregory (1973). Steps to an Ecology of Mind. Paladin Books.

Boltanski, Luc et Thévenot, Laurent (1989), « Justesse et justice dans le travail », Cahiers du centre d'études de l'emploi, Paris, Presses universitaires de France.

Callon Michel (1986), «Éléments pour une sociologie de la traduction. La domestication des coquilles Saint-Jacques et des marins-pêcheurs dans la baie de Saint-Brieuc ", L'Année sociologique, vol. 36, pp. 169-208.

Crozier, Michel (1977), L'acteur et le système, Paris, Le Seuil.

Fontaine, Nicole (2003), ministre française de l'Industrie, in Industries, n 84, mars 2003.

Foucault, Michel (1966), Les Mots et les Choses. Archéologie des sciences humaines, Paris, Gallimard, NRF.

Goffman, Erving (1959), The Presentation of Self in Everyday Life. New York: Doubleday.

Hothersall, David (1995), History of Psychology. New York: McGraw-Hill, pp. 239-253.

Latour, Bruno (1991), Nous n'avons jamais été modernes. Essai d'anthropologie symétrique. Paris, La Découverte.

Lourau, René (1990), La politique de la surimplication, Technologies et Symboliques de la Communication, sous la direction de Lucien Sfez et Gilles Coutlée, avec la participation de Pierre Musso, Presses Universitaires de Grenoble.

Maturana, Humberto \& Varela, Francisco (1980), « Autopoiesis and Cognition: The Realization of the Living », Boston Studies in the Philosophy of Science, Cohen, Robert S., \& Marx W. Wartofsky (Eds.), Vol. 42, Dordecht: Reidel Publishing Co. 
Morin, Edgar (1997), Réforme de pensée, transdisciplinarité, réforme de l'Université, Communication au Congrès International « Quelle Université pour demain ? Vers une évolution transdisciplinaire de l'Université » (Locarno, Suisse, 30 avril-2 mai 1997) ; texte publié dans Motivation, $n^{\circ} 24,1997$.

Nonaka, Ikujiro \& Takeuchi, Hirotaka (1995), The Knowledge-Creating Company, Oxford University Press.

Orléan, André (Ed.), (2000), Analyse économique des conventions, Paris, PUF. 